\title{
Simplified Model of Enterocutaneous Fistula in the Rat
}

\section{Camargo $\mathrm{CP}^{2}$, Kubrusly $\mathrm{MS}^{1}$, Lee $\mathrm{AD}^{1}$, Traldi $\mathrm{MC}^{3}$, Maluf FC, Costa $\mathrm{AC}^{4}$, Figueira ERR, Gemperli ${ }^{2}$, Carneiro-D'Albuquerque LA ${ }^{1}$ and Galvão $\mathrm{FH}^{1 *}$}

${ }^{1}$ LIM 37, Laboratorio de Transplante e Cirurgia de Figado, Departamento de Gastroenterologia, Hospital das Clinicas HCFMUSP, Faculdade de Medicina, Universidade de Sao Paulo, Sao Paulo, SP, Brazil

${ }^{2}$ LIM 04, Laboratorio de Microcirurgia e Cirurgia Plastica, Hospital das Clinicas HCFMUSP, Faculdade de Medicina, Universidade de Sao Paulo, Sao Paulo, SP, Brazil ${ }^{3}$ Resident of Departamento de Cirurgia, Hospital das Clinicas HCFMUSP, Faculdade de Medicina, Universidade de Sao Paulo, Sao Paulo, SP, Brazil

${ }^{4}$ Departamento de Patologia, Hospital das Clinicas HCFMUSP, Faculdade de Medicina, Universidade de Sao Paulo, Sao Paulo, SP, Brazil

*Corresponding Author: Galvão FH, LIM 37, Laboratorio de Transplante e Cirurgia de Figado, Departamento de Gastroenterologia, Hospital das Clinicas HCFMUSP, Faculdade de Medicina, Universidade de Sao Paulo, Sao Paulo, SP, Brazil.
Received: October 18, 2021

Published: December 09, 2021

(C) All rights are reserved by Galvão FH., et al.

\begin{abstract}
Introduction: Enterocutaneous fistula (ECF) is a hazardous postoperative complication in abdominal surgeries that demand costly treatment and can reach a mortality incidence as high as $30 \%$. There is a lack of animal models of ECF to test new strategies treatment. In this study we describe in detail an efficient and simplified model of ECF.

Methods: After general anesthesia, thirteen male Lewis rats were submitted to a midline incision. The cecum was identified and exteriorized through a small incision in the right flank to create the enterocutaneous fistula. The animals were observed twice a week searching for clinical signs of complications (abdominal distention, piloerection, infection, herniation, necrosis or fistula) and body weight assessment. At the $30^{\text {th }}$ post-operative day (POD), the animals were euthanized to perform autopsy, evaluating abdominal cavity and collecting fistula samples for histology (HE).

Results: One animal was sacrificed at the $2^{\text {nd }}$ POD because intestinal prolapse. The remaining 12 animals lost around $10 \%$ of the original weight, presented signs of moderate clinical stress but survived untill $30^{\text {th }}$ POD with a steady stercoral fistula and continuous feces output. Histological analysis confirmed the diagnosis of fistula, observing an important lymphomononuclear component in subserous layer and ulceration of squamous epithelium in the fistula border.
\end{abstract}

Conclusion: This simplified model was effective to create a consistent enterocutaneous fistula with continuous output during 30 days.

Keywords: Intestine; Fistula; Model; Rat

Citation: Galvão FH., et al. "Simplified Model of Enterocutaneous Fistula in the Rat". Acta Scientific Gastrointestinal Disorders 5.1 (2022): 06-10. 


\section{Introduction}

Enterocutaneous fistula (ECF) is an abnormal connection between the gastrointestinal (GI) tract and the skin. Any surgical intervention creating an anastomosis in the digestive tract may cause leakage resulting in an ECF [1,2]. Indeed, gastrointestinal surgery is responsible for $80 \%$ of all ECF cases, achieving a mortality rate ranging from $6 \%$ to $33 \%$. Spontaneous fistula in non-trauma and non-emergency surgeries occur more commonly in inflammatory bowel disease (IBD), but also in malignancy, appendicitis, diverticulitis, radiation, tuberculosis/actinomycosis, and ischemia [1-3].

Trauma victims also represent a group of patients that can develop post-operative ECF with an incidence around $2 \%$. In this situation, ECF etiology is related to damage control strategies and hemodynamic instability. Fisher., et al. [4]. showed 1.9\% of ECF in 2,224 patients with abdominal trauma. The incidence of ECF was higher in open ( $8 \%$ ) than closed $(0.5 \%)$ abdomen trauma. Low rate of spontaneous closure was observed in both groups. Hatchimonji., et al. [5]. reported that 135,595 patients submitted to emergency general surgery reached $1.1 \%$ of ECF incidence with high mortality rate $(10.1 \%)$.

ECF usually arises 7 to 10 days after surgery, initiating with a wound infection, followed by leakage of enteric contents through the abdominal wall. The leakage arises through the prior surgical wound incision or nearby. The main clinical signs of ECF include cutaneous rash around the fistula, malnutrition, electrolyte disturbances, dehydration, metabolic instabilities, infection and prolonged hospital stays. Most deaths are related to sepsis (77\%), malnutrition and fluid/electrolyte loss $[1,2]$. The closure rate without surgery ranges from 20 to $30 \%$ and $90 \%$ of them occurs in the first month after resolution of infection or sepsis [2].

For those that fail to close spontaneously, the treatment demands prolonged hospitalization and individualized approaches. Actually, the treatment of severe ECF is costly and challenging. It demands intestinal rehabilitation services with multidisciplinary attendance, including wound care, home parenteral nutrition and eventually complex surgical procedures such as intestinal reconstructions and/or transplantation [2,6-9].

The rat is a crucial model to study ECF and start therapeutic strategies aiming clinical applications. Hospital das Clinicas da
Faculdade de Medicina da Universidade de São Paulo (HCFMUSP) is the reference service in Brazil to treat complex GI fistulas using advanced procedures such intestinal and multivisceral transplantation $[10,11]$. Our laboratory performs rat models to develop new strategies to treat GI fistula, including intestinal and multivisceral transplantation [12-16]. The idea is to perform intestinal transplantation in rats with fistula to simulate a genuine clinical situation (translational research). The aim of this study was to describe in detail a simplified and efficient model of ECF.

\section{Material and Methods}

Thirteen isogenic SPF Lewis rats weighting 200 to 250 grams were maintained in the controlled laboratory of medical investigation 37 animal's facility from HCFMUSP. The animals were kept in a meticulously controlled environment specific for SPF rats experiments containing standard atmosphere parameters (silent, temperature of $22 \pm 2$, humidity of $55 \pm 5 \%, 12 / 12 \mathrm{~h}$ day/night cycles, absolute filtered air flow, constant natural barometric pressure) water and food was accessible ad libidum and the surgeries performed in the microsurgical room. This study was approved by our institutional animal ethics committee (\# 1278/19). The study followed ethical principles of animal research according to the National Council of Animal Experimentation

\section{Experimental design}

The rats were submitted to the surgical procedure to create the ECF and observed twice a week for body weight and clinical examination. The experimental end point was survival at $30^{\text {th }} \mathrm{POD}$, when the animal was euthanized for autopsy. Animals presenting complex complication such loss of more than $25 \%$ of the initial body weight stress posture and/or moribund state were euthanized prematurely for diagnosis.

\section{Surgical procedures}

Under inhaled anesthesia with isoflurane (isoflorano, Cristália ${ }^{\circledR}$, Sao Paulo, Brazil ) $100 \mathrm{ml} / \mathrm{min}$, the rats were submitted to a midline incision of $50 \mathrm{~mm}$ and a small right flank incision, $15 \mathrm{~mm}$ far from de midline. The intestine was exposed through the midline incision (Figure $1 \mathrm{~A}$ ), the cecum was identified and its proximal part brought out by the flank incision through the subcutis (figure 1B). The external cecum layer was sutured in the intraperitoneal abdominal layer by five stitches using polipropilene 5-0 (Prolene Eth- 


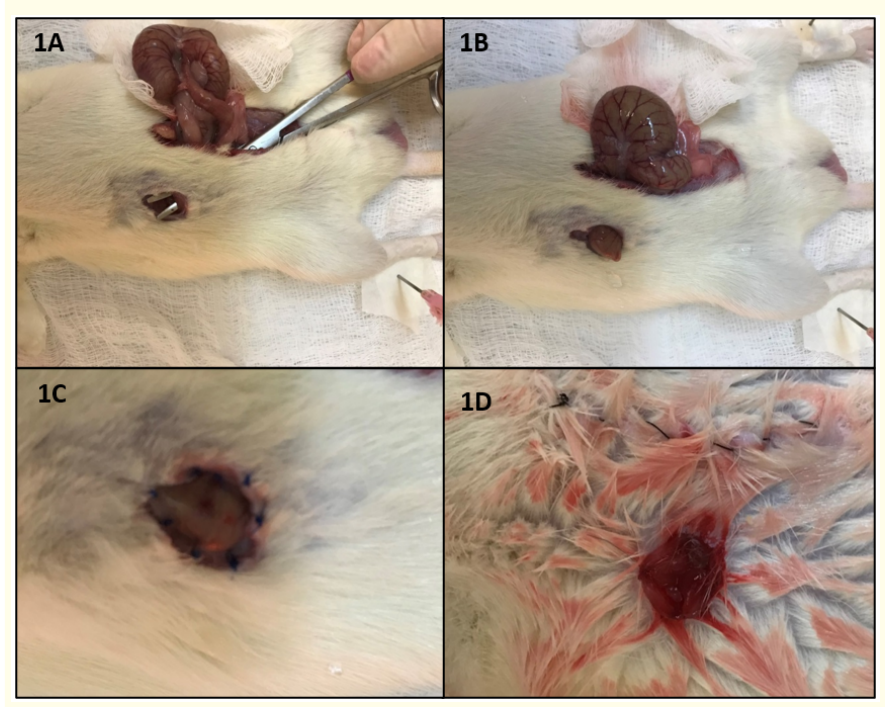

Figure 1: Details of the surgical technique. 1A- Midline incision of $50 \mathrm{~mm}$ and a small right flank incision of $15 \mathrm{~mm}$. 1B- The cecum was identified and its proximal part was pulled throughout the flank abdominal incision. 1C- The upper extremity of the cecum, and the external border was fixed by five stitches in the subcutis layer using mono nylon 5-0 (Ethicon, USA) and finally fixed in the skin. 1D-Abdominal wall closure using a continuous suture in the muscular layer and skin.

icon, USA). Additionally, we cut the upper extremity of the cecum, and the external border was fixed by five stitches in the subcutis layer using mono poliamide 5-0 (nylon, Ethicon, USA). After a circular incision of the exteriorized cecum, its border was finally fixed in the skin (figure 1C). Subsequently, we closed the abdominal wall using a continuous suture of nylon 4-0 (poliamide, Ethicon, Cincinnati, OH, USA) in the muscular layer and skin (Figure 1D).

At the experimental end point, the animals were anesthetized by intraperitoneal injection of ketamine $100 \mathrm{mg} / \mathrm{kg}$ (Ketamin, Cristalia, Sao Paulo, Brazil) combined with xylazine $10 \mathrm{mg} / \mathrm{kg}$ (Rompun, Bayer, Sao Paulo, Brazil) and placed in a euthanasia chamber with $\mathrm{CO}_{2}$. During the autopsy we investigated the macroscopic abdominal cavity condition and removed the fistula segment for histologic assessment.

\section{Post-operative evaluation}

The animals were observed twice a week searching for clinical signs of complications (abdominal distention, piloerection, fistula aspect and debit, local infection, dehiscence, necrosis, prolapses, and herniation) and body weight measurement.

\section{Histological analysis}

Samples of the fistula segment were prepared for microscopic analysis. The samples were immersed in a fixative solution of $4 \%$ phosphate-buffered paraformaldehyde for 12 hours and then submitted for processing with hematoxylin-eosin (HE) staining.

\section{Results}

The surgical procedure was quite simple for basic residents of surgery and could be totally performed within a period of 15 minutes by a single surgeon. One animal was sacrificed in the $2^{\text {nd }}$ POD due to intestinal prolapse. This complication, motivate a slight modification in the original surgical procedure by adding three internal stiches to fix it between the abdominal internal aponeurosis adjacent to the right flank incision and the intestine. After this modification there was no longer prolapse in any animals. The remaining 12 animals survived until the experimental end point. All animals lost around $10 \%$ of its original weight within the fourth POD and did not recovery it, keeping the same weight until the experiment end point. All animals presented progressive signs of clinical stress due to its filth condition with piloerection, conjunctivitis, and aggressive posture. There was a continuous leakage of intestinal contents by the fistula causing animal's filth condition and promoting a progressive cutaneous rushes and fur loss around the fistula and upper abdomen (Figure 2). The histological analysis confirmed the diagnosis of fistula observing transmural edema, involving the structures of the intestinal wall, associated with an exuberant inflammatory reaction of lymphomononuclear cells, forming lymphoid wall aggregates, ulcerations of the squamous epithelium, associated with the underlying inflammatory and vascular reaction, similar to the finds of fistula in clinical patients (Figure 3).

\section{Discussion}

Surgical models in animals are frequently needed; however, there are few articles in the literature demonstrating convenient 


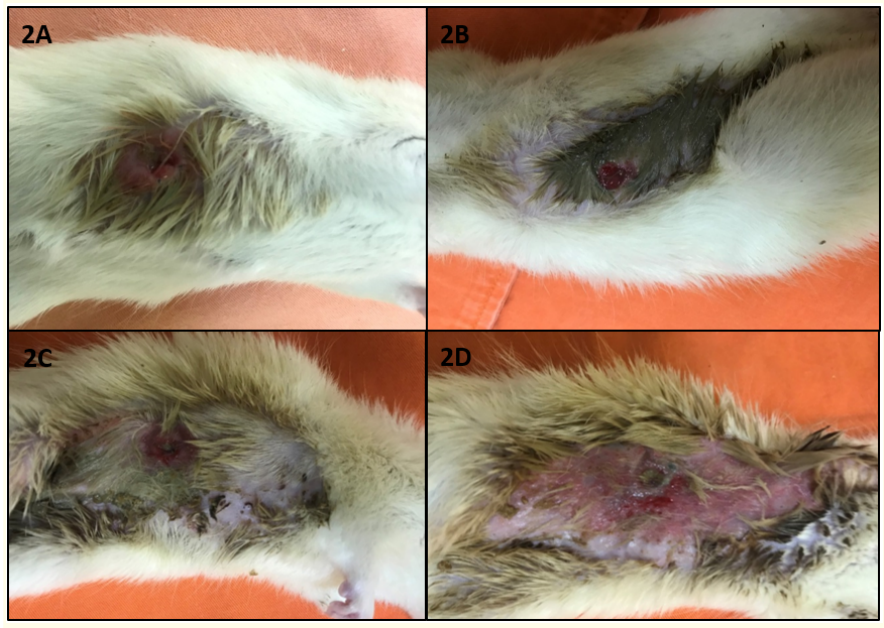

Figure 2: Pictures of the animal's evolution showing animal clinical condition (piloerection) and the progressive cutaneous rush around the fistula and abdomen at $7^{\text {th }}(2 \mathrm{~A}), 14^{\text {th }}(2 \mathrm{~B}), 21^{\text {th }}(3 \mathrm{C})$ and $30^{\text {th }}$ (2D) POD.

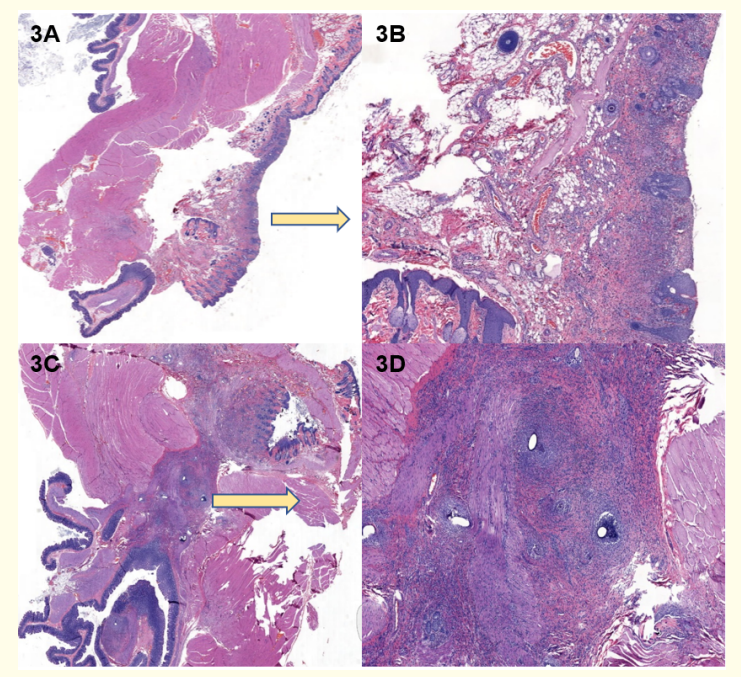

Figure 3: Panoramic micrograph of the histological section (HE $100 \mathrm{X}$ ), showing an ulcerated area of the mucosa with submucosal edema, discontinuity of mucosal epithelial layer, edema and an exuberant inflammatory reaction. 1b: High magnification micrograph (HE $400 \mathrm{X}$ ) showing, in detail, an inflammatory infiltrate in the ulcerated area. 1c: Panoramic micrograph of histological section showing the fistulous path with edema and an exuberant inflammatory reaction and involvement in muscular and submucosal structures by inflammatory process. Figure 1d. Micrograph of the fistulous path in high magnification (HE $400 \mathrm{X}$ ), showing in detail the infiltrate involving submucosal structures.
ECF simulations. Rats are very sensitive to gastrointestinal fistula or stomas. In an experiment of our group using anorectal resection and colostomy, we observed high rate of weight loss, diarrhea, cachexia, with a high mortality rate (58.3\%), compared to the good post-operative evolution of rats submitted to anorectal autotransplantation, even when the graft was submitted to a heterotopic anal implantation [13]. Wittig., et al. [18] also observed bad evolution in a rat model of loop colostomy with a $30 \%$ postoperative mortality rate. Rat models of gastric and small intestine fistula have also been proposed; however, the rats presented spontaneous fistula closure [17] or high mortality rate related to hydroelectrolytic and metabolic disturbances caused by high fistula output and infections $[18,19]$.

The ideals characteristics for an ECF model are: one-stage and straightforward procedure, low postoperative mortality rate, no spontaneous fistula closure, direct access to the fistula, low costs, controlled discomfort for animals and long-term survival rate. Considering all these requirements and based on previous animal models in rats, our group suggest in this experiment the use of the cecum for ECF, because its natural mobility and the convenient controlled output of intestinal content throughout the fistula that can be placed in the upper abdominal flank, out of rat's mouth reach.

This surgical fistula model is relatively simple to be performed even by elementary residents. Furthermore, the fistula debit is not high to cause animal mortality, but consistent enough to keep it opened over time. Therefore, in this experiment the animals survived with a functional fistula until the experimental end point, with a low rate of surgical failure $(8.3 \%)$.

\section{Conclusion}

In this experiment we showed in details an effective, simple and steady model of enterocutaneous fistula, similar to that observed in clinical trials.

This model may be a good tool to test innovative fistula treatments including reconstructive surgeries, wound care devices such percutaneous aspiration and drainage curatives and stem cells therapy.

\section{Bibliography}

1. Berry S M and Fischer J E. "Classification and pathophysiology of enterocutaneous fistulas". Surgical Clinics of North America 76.5 (1996): 1009-1018.

2. Gribovskaja-Rupp I and Melton GB. "Enterocutaneous Fistula: Proven Strategies and Updates”. Clinics in Colon and Rectal Surgery 29.2 (2016): 130-137. 
3. Wercka J., et al. "Epidemiology and outcome of patients with postoperative abdominal fistula". Revista do Colégio Brasileiro de Cirurgiões 43.2 (2016): 117-123.

4. Fischer PE., et al. "A ten-year review of enterocutaneous fistulas after laparotomy for trauma". Journal of Trauma 67.5 (2009): 924-928.

5. Hatchimonji JS., et al. "Enterocutaneous fistula after emergency general surgery: Mortality, readmission, and financial burden". The Journal of Trauma and Acute Care Surgery 89.1 (2020): 167-172.

6. Quinn M., et al. "Management of Enterocutaneous Fistula: Outcomes in 276 Patients". World Journal of Surgery 41 (2017): 2502-2511.

7. Winder J S and Pauli E M. “Comprehensive management of fullthickness luminal defects: the next frontier of gastrointestinal endoscopy". World Journal of Gastrointestinal Endoscopy's 7.8 (2015): 758-768.

8. Avalos-González J., et al. "Reduction of the closure time of postoperative enterocutaneous fistulas with fibrin sealant". World Journal of Gastroenterology 16.22 (2010): 2793-2800.

9. Huang JJ., et al. "3D-printed "fistula stent" designed for management of enterocutaneous fistula: An advanced strategy". World Journal of Gastroenterology 23.41 (2017): 7347-7494.

10. Lee ADW., et al. "Home parenteral nutrition program and referral of potential candidates for intestinal and multivisceral transplantation in a single Brazilian center". Transplantation Proceedings 46 (2014): 1839-1841.

11. Galvão FH., et al. "Experimental animal model of graft-versushost disease (GVHD) after small-bowel transplantation: characteristics of the model and application to developing treatment strategies". Transplantation Proceedings 29.1-2 (1997): 700 .

12. Galvão FH., et al. "Anorectal transplantation". Techniques in Coloproctology 13.1 (2009): 55-59.

13. Galvão FH., et al. "Simplified rat model of intestinal transplantation”. Transplantation 80.10 (2005): 1522-1523.

14. Galvão FH., et al. "Modified multivisceral transplantation in the rat". Transplantation 96.2 (2013): e3-4.
15. Galvão FH., et al. "Allogeneic anorectal transplantation in rats: technical considerations and preliminary results". Scientific Report 6 (2016): 30894.

16. Bültmann O., et al. "Creation of an enterocutaneous fistula". Research in Experimental Medicine (Berl) 198.4 (1998): 215-228.

17. Wittig G., et al. "Der Einfluß der Ingesta auf die Kanzerisierung des Rattendarmes durch Dimethylhydrazin". Arch Geschwulstforsch 37 (1971): 105-115.

18. Kohn M. "Satiation of hunger from stomach versus mouth feeding". Journal of Comparative and Physiological Psychology 44 (1951): 412-422.

19. Bleyl DWR., et al. "Zur Methodik der Ileostomie bei der Laborratte". Z Versuchstierkd 23 (1981): 46-51.

\section{Assets from publication with us}

- Prompt Acknowledgement after receiving the article

- Thorough Double blinded peer review

- Rapid Publication

- Issue of Publication Certificate

- High visibility of your Published work

Website: www.actascientific.com/

Submit Article: www.actascientific.com/submission.php

Email us: editor@actascientific.com

Contact us: +919182824667 